\title{
Troca de Valva com a Prótese Valvar Modelo Omnicarbon. Seguimento Clínico de 10 Anos
}

\author{
Valve Replacement with the Omnicarbon Valve Prosthesis. A 10-Year Follow-Up
}

Santiago Florez, Salvatore Di Stefano, Yolanda Carrascal, Juan Bustamante, Enrique Fulquet, José Ramón Echevarria, Casquero Elena, Javier Gualis, Luis Fiz

Valladolid, Espanha

\section{Objetivo}

Estudar retrospectivamente os resultados de 264 pacientes submetidos à implementação cirúrgica de válvula modelo Omnicarbon entre abril 1985 e maio 1995.

\section{Métodos}

No momento da cirurgia, a média de idade dos pacientes que receberam essa prótese mecânica era de $57 \pm 11$ anos. As válvulas modelo Omnicarbon foram colocadas em posição aórtica em $36 \%$ dos casos, na posição mitral em $44 \%$ dos casos, e nas duas posições em $20 \%$ dos casos. O seguimento clínico foi feito cuidadosamente, com a maioria dos pacientes submetidos ao exame físico em nossa clínica. Levando em conta o histórico do caso, os cardiologistas faziam perguntas aos pacientes sobre as complicações relacionadas à válvula.

\section{Resultados}

O seguimento acumulado dos pacientes foi de 1291 anos, com média de seguimento de 5,4 anos. A sobrevida após 10 anos foi de $79,4 \pm 3,9 \%$, incluindo todas as causas de morte e os casos de mortalidade precoce. As complicações relatadas durante os 11 anos de estudo incluem: tromboembolismo $(0,1 \%)$, hemorragia $(0,4 \%)$, endocardite $(0,2 \%)$, e insuficiência não-estrutural $(1,2 \%)$. Não foram detectadas anemia hemolítica, trombose valvar, ou insuficiência estrutural durante esse longo período de estudo. A capacidade funcional desses pacientes foi avaliada subjetivamente pelo sistema de classificação da NYHA. Com o tempo de seguimento maior do que 5 anos em média, nossos pacientes que receberam a válvula modelo Omnicarbon se encontram na classe I ou II da NYHA.

\section{Conclusão}

As próteses mecânicas modelo Omnicarbon apresentam um bom desempenho clínico por até 10 anos, tanto em posição aórtica quanto mitral. Os resultados indicam uma baixa incidência de tromboembolismo e complicações hemorrágicas.

\section{Palavras-chave}

válvula cardíaca mecânica, morbidade, sobrevida, tromboembolismo

\section{Objective}

We retrospectively examined the outcomes of 264 patients who underwent consecutive Omnicarbon valve implantation surgery between April 1985 and May 1995.

\section{Methods}

At the time of surgery, patients who received this mechanical prosthesis averaged $57 \pm 11$ years of age. Omnicarbon valves were placed in the aortic position in $36 \%$ of the cases, in the mitral position in $44 \%$, and in both positions in $20 \%$. Follow-up was carefully performed, with most patients undergoing physical examination at our clinic. While taking the case history, cardiac physicians specifically questioned the patient about valve-related complications.

\section{Results}

Accumulated total patient-years is 1291 , with a mean followup time of 5.4 years. Survival at 10 years is $79.4 \pm 3.9 \%$, including all causes of death and early mortality. Complications recorded during the 11-year study include: thromboembolism $(0.1 \%)$, hemorrhage $(0.4 \%)$, endocarditis $(0.2 \%)$, and nonstructural failure (1.2\%). No hemolytic anemia, valve thrombosis, or structural failure was detected during this long-term experience. Functional capability of these patients was subjectively assessed by the NYHA classification system. With follow-up time averaging over 5 years, $97 \%$ of our Omnicarbon valve patients are in NYHA I or II.

\section{Conclusion}

The Omnicarbon mechanical prosthesis provides a good clinical performance for up to 10 years in both the aortic and mitral positions. Results indicated a low incidence of thromboembolism and of hemorrhagic complications.

\section{Key words}

mechanical heart valve, morbidity, survival, thromboembolism

Instituto de Ciencias del Corazon (ICICOR), Servicio de Cirugía Cardíaca. Hospital Universitario de Valladolid, Spain 
Desde de que Hufnagel implantou a primeira prótese valvar na aorta descendente em 1960, avanços tecnológicos contínuos foram feitos na estrutura e no design para obter a válvula cardíaca artificial ideal. Entretanto, os avanços obtidos na engenharia biomédica nem sempre refletem uma melhora nos resultados clínicos. Nesse sentido, consideramos os estudos retrospectivos com seguimento clínico de longo prazo para avaliar as melhorias técnicas, particularmente interessantes do ponto de vista clínico.

Nosso departamento começou a utilizar a válvula de Omnicarbon (Medical Incorporated, Minneapolis, USA) em 1985. 0 presente estudo selecionou pacientes submetidos à cirurgia de abril de 1985 a maio de 1995. Nosso estudo foi baseado nas diretrizes publicadas por Edmunds para descrever eventos de mortalidade e morbidade ${ }^{1}$.

A válvula de Omnicarbon é uma válvula de disco lenticular simples feita de carbono pirolítico, com dois pivôs que se estendem da armação feita do mesmo material. A válvula é montada em um anel de sutura muito flexível feito de teflon sem costuras. É importante enfatizar a facilidade de adaptação do anel de sutura ao anel original, e também que é possível virar a armação da válvula².

\section{Métodos}

Um total de 318 próteses valvares modelo Omnicarbon foram implantadas em 264 pacientes no Hospital Clínico Universitário de Valladolid. Essa população foi distribuída igualmente entre 133 homens $(50,4 \%)$ e 131 mulheres $(49,6 \%)$. A idade media no momento da cirurgia foi $56,7 \pm 11,2$ anos, com idades variando de 24 a 80 anos.

Houve 95 (36\%) cirurgias de válvula aórtica (CVA), 115 (44\%) mitral (CVM), e 54 (20\%) casos com cirurgia nas duas válvulas (CDV). 0 tamanho da válvula (diâmetro do anel) mais utilizado na posição aórtica foi 25 , e 29 na posição mitral (fig.1)

Oitenta e nove cirurgias foram previamente realizadas em 67 pacientes (25\%) incluindo 35 trocas de válvula, 26 comissurotomias, 14 anuloplastias, 12 implantes de marcapasso, e 2 outras intervenções cardíacas. No momento da cirurgia, 66\% (174/264) dos pacientes apresentavam classe III ou IV da NYHA, 32\% (84/ $264)$ estavam na classe II, e apenas $2 \%$ (6/264) na classe I. Cirurgia eletiva foi feita em $89 \%$ dos casos (235/264), e as outras $11 \%(29 / 264)$ foram cirurgias de emergência.

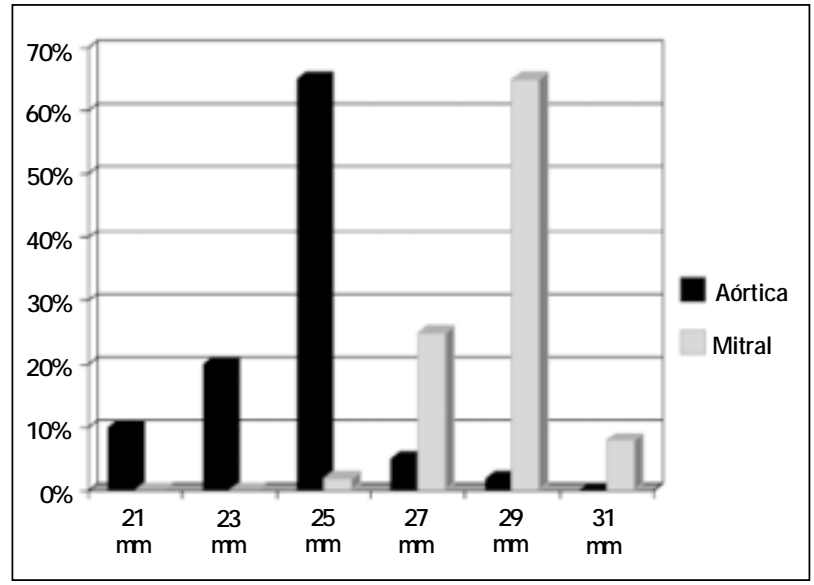

Fig. 1 - Tamanho da válvula em posição aórtica e mitral.
A cirurgia foi feita com circulação extracorpórea e hipotermia sistêmica moderada. No geral, a prótese valvular mitral foi orientada com o orifício maior direcionado para a via saída do ventrículo esquerdo. As próteses aórticas foram orientadas com a movimentação do disco em direção anterior direita. Nossa técnica de sutura usou em sua maioria as suturas interrompidas de linha de poliéster apoiadas com almofadas de teflon (pledgets).

Sistematicamente, a anticoagulação foi iniciada com heparina sódica até que fosse alcançada a proteção efetiva com dicumarínicos. A anticoagulação oral com dicumarínicos foi iniciada quando o dreno foi removido, e foi mantida indefinidamente. 0 potencial tromboembolítico valvular pós-operatório foi individualizado, com ênfase especial no período de risco máximo representado pelas primeiras horas ou dias após a cirurgia. Nesse sentido, é benéfico o tratamento rápido com heparina simultaneamente com os anticoagulantes orais até que o paciente alcance o nível desejado de anticoagulação. A associação de dicumarínicos com dipiridamol foi utilizada em pacientes com risco elevado de complicações tromboembolíticas, como por exemplo, na fibrilação atrial e história prévia de eventos tromboembólicos. A dose de dicumarínicos foi ajustada respeitando-se o tempo de protrombina (TP) expresso em percentagem de atividade (25-35\%) ou, em anos mais recentes de acordo com a razão normalizada internacional (RNI). A terapia com anticoagulante foi ajustada para cada paciente para manter a RNI entre 2,5 e 3,5 em pacientes na (CDV) ou na (CVM) e entre 2,0 e 3,0 nos pacientes de (CVA).

O seguimento clínico foi ambulatorial para a maioria dos pacientes (180/226 pacientes, 80\%), por telefone em 26 pacientes (12\%), por carta em 6 pacientes (3\%), e em 14 pacientes (6\%) foi feito através da revisão dos registros clínicos. Foi impossível contatar 13 pacientes, portanto esse estudo de 11 anos teve informação do seguimento clínico para 95,1\% dos pacientes.

O seguimento clínico máximo foi de 11,1 anos com média de 5,4 0,2 anos, com um total de 1291 paciente-anos, incluindo 239 pacientes em risco (CVA: 491, CVM: 546, e CDV: 254).

O seguimento ecocardiográfico foi feito com ecocardiograma transtorácico convencional. Quando os ecocardiografistas encontravam uma anomalia, a ecocardiografia transesofágica era feita para definir o problema.

A classificação das causas de morbidade e mortalidade foi feita seguindo as diretrizes publicadas pela Society of Thoracic Surgeons (Sociedade de Cirurgiões Torácicos) e American Association for Thoracic Surgery (Associação Americana de Cirurgia Torácica) ${ }^{1}$. Os dados foram expressos quando apropriados como média \pm desvio padrão, porcentagens simples, ou razão linear de eventos com erro padrão.

Todas as curvas de sobrevida e curvas livres de eventos foram calculadas utilizando o método atuarial e as probabilidades estão expressas com o erro padrão.

\section{Resultados}

Vinte e cinco pacientes morreram durante o pós-operatório (<30 dias ou antes da alta hospitalar), a taxa de mortalidade precoce foi de $9,5 \%(4,2 \%$ CVA, 12,2\% CVM, 13\% CDV). A idade media desse grupo foi $59,6 \pm 13,2$ anos, significativamente maior do que idade média de todos os pacientes $(p<0,001)$.

A causa mais comum de morte foi baixo débito cardíaco (7/25), 
seguido por falência de múltiplos órgãos em 6 casos, hemorragia não-controlada em 3 casos, arritmia ventricular maligna em 2 casos, embolia cerebral em 1 caso, infarto agudo do miocárdio em 1 caso, septicemia generalizada em 1 caso. Os 4 pacientes restantes morreram de outras causas não-cardíacas.

Trinta pacientes (9 CVA, 14 CVM, 7 CDV) morreram após sobreviverem ao pós-operatório, levando a uma taxa linear de $2,3 \pm 0,4 \%$ /paciente-ano $(1,8 \pm 0,6 \%$ para CVA, $2,6 \pm 0,7 \%$ para CVM e $2,8 \pm 1,0 \%$ para CDV). Onze pacientes morreram devido a complicações relacionadas à válvula, de acordo com a definição de Edmunds ${ }^{1}$ : morte súbita (5), hemorragia (2), endocardite (2), e extravasamento perivalvular (2). 0 mesmo número de pacientes (11/30) morreram de doenças ligadas a causas cardíacas: 10 por insuficiência cardíaca e 1 por arritmia. A causa de morte de 3 pacientes é desconhecida e nos outros 5 pacientes a morte foi atribuída a causas não-cardíacas.

A sobrevida total em 10 anos, considerando todas as causas de morte e incluindo mortalidade precoce, é de $79,4 \pm 3,9 \%$ $(81,2 \pm 6,4 \%$ em CVA, $78,5 \pm 5,5 \%$ em CVM, e $78,8 \pm 7,5 \%$ em $\mathrm{CDV}$ ). As curvas de sobrevida de acordo com cada grupo de pacientes se encontram na figura 2 . Se apenas a mortalidade tardia associada à troca valvar for considerada, a sobrevida em 10 anos é de $95,5 \pm 1,8 \%(95,7 \pm 2,5 \%$ na CVA, $90,2 \pm 4,4 \%$ na CVM, e $93,3 \pm 4,6 \%$ na CDV).

Durante o período de seguimento clínico, a curva atuarial livre de eventos tromboembolíticos em 10 anos foi de $99,5 \pm 0,5 \%$ $(97,6 \pm 2,4 \%$ para CDV). Apenas um paciente apresentou evento tromboembolítico (CDV, não-fatal). A taxa linear é de $0,1 \pm 0,1 \%$ / pta $(0,4 \pm 0,4 \% / p t a C D V)$. Não houve casos de trombose valvar.

A taxa linear para hemorragia é de $0,4 \pm 0,2 \% / p t a(0,6 \pm 0,4 \% /$ pta CVA e $0,8 \pm 0,6 \% / p t a C D V)$. A sobrevida atuarial livre de eventos hemorrágicos em 10 anos foi de $96,1 \% \pm 2,5(94,2 \pm 4,3 \%$ em CVA e $92,3 \pm 3,1 \%$ em CVD). Cinco acidentes sérios relacionados ao uso de anticoagulantes ocorreram em 3 pacientes (2 CVA, 1 CVD). Em 3 ocasiões, a hemorragia teve origem digestiva; houve também um caso fatal de hemorragia intracraniana e um sangramento urológico não fatal. Os dois pacientes que morreram de hemorragia tiveram diagnóstico prévio de extravasamento aórtico perivalvular com repercussão clínica e estavam aguardando a reoperação. 0 terceiro paciente (CDV) que apresentou sangramento também apresentava hipertensão pulmonar severa e insuficiência ventricular direita com deterioração hepática secundária, necessi-

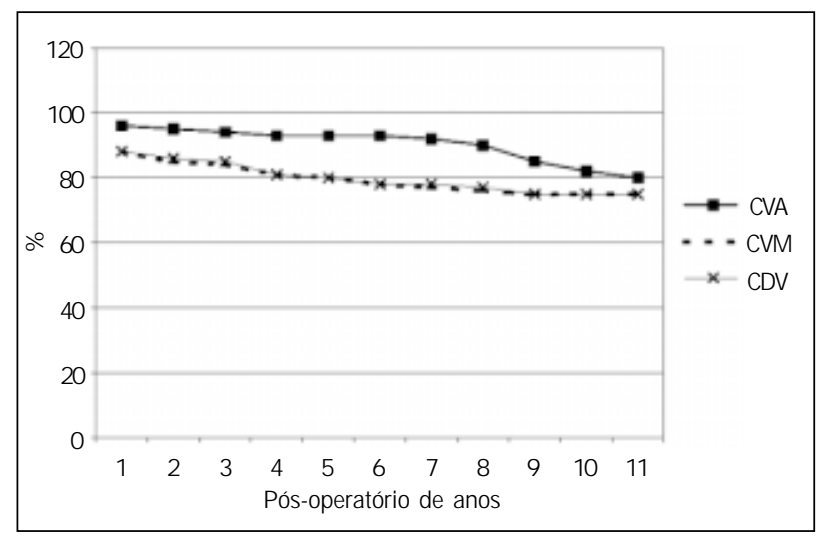

Fig. 2 - Sobrevida. tando hospitalizações devido ao sangramento digestivo e hematúria. Esse paciente morreu 2 anos após a cirurgia realizada com a finalidade de melhorar a insuficiência cardíaca.

A incidência combinada de tromboembolismo e hemorragia é de $0,5 \pm 0,2 \% /$ pta.

Foram diagnosticados dois casos de endocardite da prótese no pós-operatório tardio (CVM, 1 CVD). Isso corresponde a uma taxa linear de $0,2 \pm 0,1 \% /$ pta $(0,2 \pm 0,2 \% /$ pta em CVM e $0,4 \pm 0,4 \% / p t a$ em CVD). Os dois pacientes foram reoperados, trocando as válvulas infectadas, e ambos morreram durante 0 pós-operatório. A sobrevida atuarial livre de endocardite em 10 anos foi de $98,0 \pm 0,6 \%(98,2 \pm 1,3 \%$ para CVM e $96,1 \pm 2,1 \%$ para CDV).

Deficiência não-estrutural foi diagnosticada de acordo com os dados da ecocardiografia transtorácica, e conforme dito na seção material e métodos, a ecocardiografia transesofágica foi realizada quando os ecocardiografistas precisavam definir o problema. As decisões terapêuticas foram feitas utilizando os critérios padrões, de acordo com os dados ecocardiográficos e a situação clínica (envolvimento hemodinâmico e anemia hemolítica).

As deficiências não estruturais foram detectadas na forma de deiscência valvular ou crescimento do pannus (pannus é constituído por tecido sinovial hiperplasiado e hipertrofiado) em 15 pacientes (6 CVA, 6 CVM, 3 CDV). Correspondente a uma taxa linear de $1,2 \pm 0,3 \% /$ pta $(1,2 \pm 0,5 \% /$ pta para CVA, $1,1 \pm 0,4 \% /$ pta para MYR, e $1,2 \pm 0,7 \% /$ pta para CDV). A sobreviva atuarial livre de deficiência não estrutural em 10 anos é de $87,9 \pm 3,9 \%(89,1 \pm 5,4 \%$ para CVA, $80,7 \pm 10,3 \%$ para MYR, e $92,2 \pm 4,3 \%$ para CVD). Nesse grupo de 15 pacientes, $13(87 \%)$ apresentaram deiscência perivalvular significante com envolvimento hemodinâmico e/ou anemia hemolítica (6 CVA, 5 CVM, 2 CDV). Não foi possível corrigir cirurgicamente esse problema em 3 pacientes: 2 morreram antes da cirurgia por hemorragia severa (previamente discutida), e a cirurgia não foi considerada para o terceiro devido a situação clínica extremamente grave. Dois dos 10 pacientes remanescentes não sobreviveram à cirurgia. Episódios de deiscência corresponderam a uma taxa linear de $1,0 \pm 0,3 \% /$ pta $(1,2 \pm 0,5 \% / p t a$ em CVA, $0,9 \pm 0,4 \%$ pta em CVM, e 0,8 $\pm 0,6 \%$ /pta em CDV).

Dois pacientes (1 CVM, 1 CDV) necessitaram reoperação para corrigir um pannus periprotético. É interessante evidenciar que os dois casos apresentaram discreto (sem significância hemodinâmica) extravasamento perivalvular. A taxa linear de complicação não-estrutural é de $0,2 \pm 0,1 \%(0,2 \pm 0,2 \%$ para CVM $, 0,4 \pm 0,4 \%$ para CDV).

Exceto nos casos de extravasamento perivalvular acompanhado de hemólise, não observamos pacientes com válvula de Omnicarbon com anemia hemolítica. Além disso, não ocorreram casos de insuficiência estrutural.

Quatorze pacientes (6 CVA, 6 CVM, 2 CDV) foram submetidos a reoperação representando uma taxa linear de $11 \pm 0,3 \% / p t a$ $(I, 2 \pm 0,5 \% /$ pta para CVA, $11 \pm 0,4 \% /$ pta para CVM, e $0,8 \pm 0,6 \% /$ pta para CDV). As indicações para reoperação foram deiscência (10), pannus (2), e endocardite (2). Desse grupo de pacientes reoperados, 4 não sobreviveram à cirurgia ou morreram durante 0 pós-operatório inicial (2 por extravasamento perivalvular e 2 por endocardite). A taxa de mortalidade associada com a reoperação foi de $28,6 \%$. A sobrevida atuarial livre de reoperação em 10 anos foi de $88,7 \pm 3,9 \%(88,8 \pm 2,6 \%$ para CVA, $89,6 \pm 4,1$ para CVM, e $92,3 \pm 3,8 \%$ para CDV). 
As tabelas I e II resumem a taxa linear de complicações e as probabilidades atuariais livres de morbidade. No seguimento clínico atual (pós-operatório médio de 5,4 anos), 96,9\% dos pacientes estavam na classe I ou II da NYHA.

\section{Discussão}

A prótese mecânica de Omnicarbon ficou disponível na Espanha como uma válvula substituta em 1984. A investigação científica para produzir a terceira geração do design da omni (disco móvel e uma lateral integral) resultou na válvula de Omnicarbon; suas antecessoras foram a Lillehei-Kaster e a prótese de omniscience 2. A válvula de Omnicarbon é uma prótese simples de suporte único e feita totalmente de carbono pirolítico.

Nesse estudo retrospectivo, são analisados os resultados a longo prazo obtidos de um número significante de estudos de pacientes, 264 com 318 válvulas implantadas. 0 período de seguimento clínico (media de 5,4 0,2 anos, com um máximo de 11,1 anos) permite a avaliação do desempenho clínico da válvula de Omnicarbon a longo prazo.

A mortalidade hospitalar nessa série foi de 9,5\%, semelhante aos resultados obtidos por outros investigadores ${ }^{3-6}$. A mortalidade hospitalar está relacionada à situação funcional pré-operatoria dos pacientes, o grau de disfunção ventricular, e a idade. A principal causa da mortalidade precoce em nosso grupo foi a síndrome de baixo débito cardíaco ( $28 \%$ de todas as mortes).

\begin{tabular}{|lcccc|}
\hline \multicolumn{5}{|c|}{ Tabela I - Complicações: índices lineares \%/pta } \\
\hline Complicação & CVA & CVM & CDV & $\begin{array}{c}\text { Todos os } \\
\text { pacientes }\end{array}$ \\
\hline Tromboembolismo & 0 & 0 & $0,4 \pm 0,4$ & $0,1 \pm 0,1$ \\
Hemorragia & $0,6 \pm 0,4$ & 0 & $0,8 \pm 0,6$ & $0,4 \pm 0,2$ \\
Endocardite & 0 & $0,2 \pm 0,2$ & $0,4 \pm 0,4$ & $0,2 \pm 0,1$ \\
Falha/Defeito & $1,2 \pm 0,5$ & $1,1 \pm 0,4$ & $1,2 \pm 0,7$ & $1,2 \pm 0,3$ \\
não-estrutural & & & & \\
Anemia hemolítica & 0 & 0 & 0 & 0 \\
$\begin{array}{l}\text { Defeito estrutural } \\
\text { Reoperação }\end{array}$ & 0 & 0 & 0 & 0 \\
$\begin{array}{l}\text { Motalidade tardia, } \\
\text { todas as causas }\end{array}$ & $1,2 \pm 0,5$ & $1,1 \pm 0,4$ & $0,8 \pm 0,6$ & $1,1 \pm 0,3$ \\
\hline CVA - cirurgia valvar aórtica; CVM - cirurgia valvar mitral; CDV - cirurgia \\
de duas válvulas.
\end{tabular}

\begin{tabular}{|lccccc|}
\hline \multicolumn{5}{|c|}{$\begin{array}{c}\text { Tabela II - Complicações: probabilidades atuariais de sobrevida livre } \\
\text { de eventos em um pós-operatório de } \mathbf{1 0} \text { anos (\%) }\end{array}$} \\
\hline Complicação & CVA & CVM & CDV & $\begin{array}{l}\text { Todos os } \\
\text { pacientes }\end{array}$ \\
\hline & & & & $97,6 \pm 2,4$ & $99,5 \pm 0,5$ \\
\hline $\begin{array}{l}\text { Tromboembolismo } \\
\text { Hemorragia }\end{array}$ & 100 & 100 & $92,3 \pm 3,1$ & $96,1 \pm 2,5$ \\
$\begin{array}{l}\text { Endocardite } \\
\text { Falha/Defeito }\end{array}$ & $89,1 \pm 5,4$ & $80,7 \pm 10,3$ & $92,2 \pm 4,3$ & $87,9 \pm 3,9$ \\
estrutural & 100 & $98,2 \pm 1,3$ & $96,1 \pm 2,1$ & $98,0 \pm 0,6$ \\
Anemia hemolítica & 100 & 100 & 100 & 100 \\
$\begin{array}{l}\text { Falha/Defeito } \\
\text { estrutural }\end{array}$ & 100 & 100 & 100 & 100 \\
$\begin{array}{l}\text { Reoperação } \\
\text { Motalidade tardia, } \\
\text { todas as causas }\end{array}$ & $98,8 \pm 2,6$ & $89,6 \pm 4,1$ & $92,3 \pm 3,8$ & $88,7 \pm 3,9$ \\
\hline CVA - cirurgia valvar aórtica; CVM - cirurgia valvar mitral; CDV - cirurgia \\
de duas válvulas.
\end{tabular}

Durante o período de seguimento clínico, um total de 30 pa-

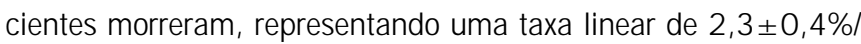
pta. Onze $(37 \%)$ devido a causas relacionadas à troca valvar (hemorragia, endocardite, disfunção não-estrutural, e morte súbita), e outros 11 pacientes morreram por razões cardíacas (principalmente insuficiência cardíaca). Portanto, a insuficiência cardíaca congestiva é considerada a causa predominante de mortalidade tardia. Nesse sentido é importante a consideração feita por NitterHauge ${ }^{7}$ que a durabilidade e a baixa trombogenicidade das válvulas mecânicas disponíveis atualmente justificam indicar a cirurgia precocemente, antes de ocorrer um dano miocárdico irreversível, melhorando assim a expectativa de vida a longo prazo ${ }^{8}$.

Após a análise estatística dos resultados, a taxa de sobrevida atuarial em 10 anos é de 95,5 $1,8 \%$, considerando apenas a mortalidade tardia associada à troca valvular. A expectativa de vida é mais baixa para os pacientes de troca de válvula mitral. Outros estudos relacionados a próteses mecânicas com tempo de seguimento clínico igual ao nosso demonstrou taxas de sobrevida semelhantes ${ }^{9-13}$.

0 tromboembolismo, que é o problema predominante relacionado às válvulas mecânicas, foi mais baixo em nossos pacientes com válvula de Omnicarbon. Não ocorreram casos de trombose da prótese. A sobrevida atuarial livre de episódios tromboembólicos é de $99,5+0,5 \%$. É óbvio que a gênese dessa complicação é multifatorial com o design da válvula sendo um componente. Nossos resultados são claramente favoráveis para a prótese de Omnicarbon e estão de acordo com outros autores que estudaram esta válvula9,10,14-17.

Nossa taxa combinada de tromboembolismo e hemorragia é baixa de $0,5 \pm 0,2 \% /$ pta $^{18}$.

A terapia antitrombótica na cirurgia de válvula cardíaca continua sendo um assunto controverso. 0 momento de iniciar a anticoagulação, o tipo de heparina a ser utilizada, os controles, a intensidade do tratamento, e a associação ou não com medicamento antiadesivo plaquetário está sendo continuamente revisado. Os resultados apresentados em nossa série podem ser uma conseqüência do esquema de anticoagulação individualizado e precoce que utilizamos. A identificação e separação de subgrupos de pacientes com riscos tromboembólicos diferentes ${ }^{19,20}$ e a padronização do protocolo de anticoagulação através da RNI permite a individualização do tratamento de anticoagulação, reduzindo o risco de hemorragia sem reduzir a proteção tromboembolítica.

Estudos prévios da prótese de Omnicarbon em outros hospitais não relataram em nenhum caso de anemia hemolítica. Knott e cols. ${ }^{21}$ do Instituto Helmholtz de Engenharia Biomédica (Alemanha) estudou as características hidrodinâmicas de 10 válvulas mecânicas diferentes, e demonstrou que a prótese de Omnicarbon apresenta uma velocidade de regurgitação e volume relativamente menor e portanto uma capacidade menor de induzir hemólise ${ }^{22}$. Nosso grupo detectou a hemólise laboratorial ou clínica apenas nas válvulas de Omnicarbon com deiscência perivalvular.

Apesar de ser completamente claro que a deiscência perivalvular e a endocardite estão diretamente relacionadas à técnica cirúrgica e/ou às circunstâncias dos pacientes mais do que ao design da válvula, essas complicações são importantes para se ter em conta quando se estiver avaliando os resultados da troca valvar. Em nosso departamento, o extravasamento periprotético é normalmente avaliado pelo exame clínico e o Eco-Doppler em cores. A literatura nesse assunto demonstra uma grande diversidade de resultados ${ }^{22-25}$, parcialmente pela variação da sensitividade 
dos diferentes métodos de diagnósticos utilizados, pela dificuldade de avaliar clinicamente a gravidade da regurgitação para incluir como uma complicação valvular, e a dificuldade de diferenciar uma regurgitação patológica de uma regurgitação transvalvular normal principalmente na presença de alta freqüência cardíaca. Como o Ecodoppler a cores tornou-se rotineiro para os pacientes com próteses valvares, a detecção de extravasamento perivalvular com graus leves ou moderados de regurgitação, é provavelmente hoje em dia, mais simples e freqüente.

O comportamento hemodinâmico da válvula de Omnicarbon demonstrou um desempenho clínico igual ao de outras válvulas mecânicas atuais ${ }^{15-17}$. Nossos dados estão de acordo com aqueles da Universidade de Bonn ${ }^{26}$ e da Universidade de Montpellier ${ }^{27}$ que estabeleceu os valores hemodinâmicos esperados para tama- nhos diferentes da válvula Omnicarbon nas posições mitral e aórtica. Saber o perfil hemodinâmico de cada válvula facilita a identificação da disfunção protética, caso esteja presente.

Consideramos uma limitação do estudo a ausência de uma análise exaustiva dos dados ecocardiográficos. Estes são importantes para avaliar a função hemodinâmica e sua repercussão na massa ventricular, e para estabelecer a exata magnitude da falha não estrutural. Nesse sentido estamos preparando um novo estudo prospectivo.

Os resultados clínicos obtidos após uma experiência de longo prazo com a válvula de Omnicarbon estabelecem essa válvula como a válvula mecânica preferida. A incidência de complicações tromboembolíticas foi notadamente baixa. A sobrevida e a qualidade de vida foram satisfatórias.

\section{Referências}

1. Edmunds J rLH, Clark RE, Cohn LH, Grunkemeier GL, Miller DC, Weisel RD. Guidelines for reporting morbidity and mortality afier cardiac valvular operations. Ann Thorac Surg 1996; 62: 932-5.

2. Bodnar E. Mechanical valves. In: Acar J, Bodnar E, eds. Textbook of Acquired Heart Valve Disease. Ist ed. London: ICR Publishers 1995: 965-1001.

3. Keenan RJ, Armitage J M, Trento A et al. Clinical experience with the Medtronic-Hall valve prosthesis. Ann Thorac Surg 1990; 50: 748-53.

4. Lopez J F, Bharadwaj B, Lal S. Experience with Hall-Kaster valves. Vascular Surg 1990; $24: 16-22$.

5. Czer LSC, ChauxA, Matloff JM et al. Ten-year experience with the St. J ude Medical valve for primary valve replacement. J Thorac Cardiovasc Surg 1990; 100: 44-55.

6. Rödler SM, M oritz A, Schreiner W, End A, Dubsky P, Wolner E. Five-year follow-up after heart valve replacement with the CarboMedics bileaflet prosthesis. Ann Thorac Surg 1997; 63: 1018-25.

7. Nitter-Hauge S. Mechanical heart valves. Conclusions from long-term follow-up. Eur Heart] 1997; 18: 907-10.

8. Iguro Y, M oriyama Y, Yamaoka A et al. Clinical experience of 473 patients with the Omnicarbon prosthetic heart valve. J Heart Valve Dis 1999; 8: 674-9.

9. Hurle A, Gomez-Plana J, Meseguer J , J uan M, Llamas P. Nine-year follow up of the Omnicarbon prosthesis in the aortic position. J Heart Valve Dis 2002; 11: 524-8.

10. Misawa Y, Fuse K, Saito T, Konishi H, Oki SI. Fourteen year experience with the Omnicarbon prosthetic heart valve. ASAIOJ 2001; 47:677-82.

11. Milano A, Bortolotti U, Mazzucco A et al. Heart valve replacement with the Sorin tilting-disc prosthesis. J Thorac Cardiovasc Surg 1992; 103: 267-75.

12. Lindblom $\mathrm{D}$, Lindblom $\mathrm{U}$, Qvist J, Lundström $\mathrm{H}$. Long-term relative survival rates after heart valve replacement. J Am Coll Cardiol 1990; 15: 566-73.

13. Akins CW. Long-term results with the Medtronic-Hall valvular prosthesis. Ann Thorac Surg 1996; 61: 806-13.

14. Thevenet A, Albat $B$. Long term follow up of 292 patients after valve replacement with the Omnicarbon prosthetic valve. J Heart Valve Dis 1995; 4: 634-9.

15. Abe T, Kamata K, Kuwaki K, Komatsu K, Komatsu S. Ten years' experience of aortic valve replacement with the Omnicarbon valve prosthesis. Ann Thorac Surg 1996; 61: 1182-7.

16. Misawa Y, Hasegawa T, Kato M. Clinical experience with the Omnicarbon prosthetic heart valve. J Thorac Cardiovasc Surg 1993; 105: 168-72.

17. Peter $M$, Weiss $P, J$ enzer $H R$ et al. The Omnicarbon tilting-disc heart valve prosthesis. J Thorac Cardiovasc Surg 1993; 106: 599-608.

18. M oriyama Y, Nakamura K, Kariyazono H, Toyohira H, Taira A. Influence of lowintensity anticoagulation and low-dose antiplatelet agent on coagulation-fibrinolysis system afier mechanical prosthetic valve replacement. J Thorac Cardiovasc Surg $1998 ; 115: 952-4$

19. Acar J, J ung B, Boissel J P et al. AREVA: Multicenter randomized comparison of low-dose versus standard-dose anticoagulation in patients with mechanical prosthetic heart valves. Circulation 1996; 94:2107-12.

20. ButchartEG, Lewis PA, Bethel J A, B reckenridge IM. Adjusting anticoagulation to prosthesis thrombogenicity and patient risk factors. Circulation 1991;84(supp III):III-61-9.

21. Knott $E$, Reul $H$, Knoch M, Steinseifer U, Rau G. In vitro comparison of aortic heart valve prostheses. J Thorac Cardiovasc Surg 1988; 96: 952-61.

22. Dumont K, Segers R, Vandenberghe S, Van Nooten G, Verdonck P. Omnicarbon $21 \mathrm{~mm}$ aortic valve prosthesis: in vitro hydrodynamic and echo-Doppler study. J Artif Organs 2002; 25: 783-90.

23. Miller DL, Monis J], Schaff HV, Mullany CJ, Nishimura RA, Orszulak TA. Reoperation for aortic valve periprosthetic leakage: Identification of patients at risk and results of operation. J Heart Valve Dis 1995;4: 160-5.

24. J indani A, NevilleEM, Venn G, Williams BT. Paraprosthetic leak: A complication of cardiac valve replacement. J Cardiovasc Surg 1991; 32: 503-8.

25. Antunes MJ . Reoperations on cardiac valves. J Heart Valve Dis 1992; 1: 15-28.

26. FehskeW, Kessel D, Kirchhoff PG, Omran H, Manz M, Lüderitz B. Echocardiographic profile of the normally functioning Omnicarbon valve. J Heart Valve Dis 1994;3:263-74.

27. Messner-Pellenc $P$, Wittenberg 0 , Leclercq $F$ etal. Doppler echocardiographic evaluation of the Omnicarbon cardiac valve prostheses.J Cardiovasc Surg 1993; 34: 195-202. 